\title{
A Novel Methodology for Applying Multivoxel MR Spectroscopy to Evaluate Convection-Enhanced Drug Delivery in Diffuse Intrinsic Pontine Gliomas
}

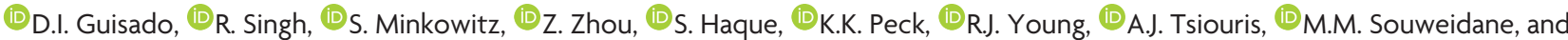 \\ (1)S.B. Thakur
}

\begin{abstract}
BACKGROUND AND PURPOSE: Diffuse intrinsic pontine gliomas are inoperable high-grade gliomas with a median survival of less than 1 year. Convection-enhanced delivery is a promising local drug-delivery technique that can bypass the BBB in diffuse intrinsic pontine glioma treatment. Evaluating tumor response is critical in the assessment of convection-enhanced delivery of treatment. We proposed to determine the potential of 3D multivoxel ' $\mathrm{H}-\mathrm{MR}$ spectroscopy to evaluate convection-enhanced delivery treatment effect in these tumors.
\end{abstract}

MATERIALS AND METHODS: We prospectively analyzed 3D multivoxel ${ }^{1} \mathrm{H}-\mathrm{MR}$ spectroscopy data for 6 patients with nonprogressive diffuse intrinsic pontine gliomas who received convection-enhanced delivery treatment of a therapeutic antibody (Phase I clinical trial NCT01502917). To compare changes in the metabolite ratios with time, we tracked the metabolite ratios Cho/Cr and Cho/NAA at several ROIs: normal white matter, tumor within the convection-enhanced delivery infusion site, tumor outside of the infused area, and the tumor average.

RESULTS: There was a comparative decrease in both $\mathrm{Cho} / \mathrm{Cr}$ and Cho/NAA metabolite ratios at the tumor convection-enhanced delivery site versus tumor outside the infused area. We used MR spectroscopy voxels with dominant white matter as a reference. The difference between changes in metabolite ratios became more prominent with increasing time after convection-enhanced delivery treatment.

CONCLUSIONS: The comparative change in metabolite ratios between the convection-enhanced delivery site and the tumor site outside the infused area suggests that multivoxel ${ }^{1} \mathrm{H}-\mathrm{MR}$ spectroscopy, in combination with other imaging modalities, may provide a clinical tool to accurately evaluate local tumor response after convection-enhanced delivery treatment.

ABBREVIATIONS: $C E D=$ convection-enhanced delivery; $\mathrm{DIPG}=$ diffuse intrinsic pontine glioma; $\mathrm{MRSI}=\mathrm{MR}$ spectroscopic imaging; preCED $=1$ week prior to $\mathrm{CED}$ treatment; postCED1 $=1$ day after CED treatment; postCED2 $=1$ month after CED treatment; postCED3 $=2$ months after CED treatment

D iffuse intrinsic pontine gliomas (DIPGs) are inoperable high-grade gliomas that account for $10 \%-15 \%$ of pediatric primary CNS tumors and $75 \%-80 \%$ of pediatric brain stem tumors. ${ }^{1,2}$ The current standard of care for DIPG is radiation therapy; despite much effort, median survival is $<1$ year. Effective

Received August 21, 2015; accepted after revision January 5, 2016.

From the Weill Medical College of Cornell University (D.I.G., R.S.), New York, NY; Departments of Radiology (S.M., A.J.T.) and Neurological Surgery (Z.Z., M.M.S.), Weill Medical College of Cornell University, New York, New York; and Departments of Radiology (S.H., K.K.P., R.J.Y., S.B.T.), Neurosurgery (M.M.S.), and Medical Physics (K.K.P., S.B.T.), Memorial Sloan Kettering Cancer Center, New York, New York.

This work was partly supported by the Memorial Sloan Kettering Cancer Center Summer Fellowship, the American Association of Neurological Surgeons Medical Student Summer Fellowship, Perry's Promise Fund, The Cristian Rivera Foundation, Solving Kids' Cancer, The Cure Starts Now Foundation, The Lyla Nsouli Foundation, the Dana Foundation, St. Baldrick's Foundation, Pediatric Brain Tumor Foundation, McKenna Claire Foundation, and lan's Friends Foundation. This research was partially supported by the Memorial Sloan Kettering Cancer Center Support Grant/ Core Grant (P30 CA008748).

Memorial Sloan Kettering has exclusively licensed the $8 \mathrm{H} 9$ used in this trial to Y-mAbs Therapeutics. drug delivery to these brain tumors is an obstacle. ${ }^{3}$ The relatively intact blood-brain barrier presents a major hurdle in drug delivery to DIPGs. Convection-enhanced delivery (CED) is a local drug-delivery technique that bypasses the $\mathrm{BBB}$ and uses hydraulic pressure to deliver agents directly to the brain tumor. CED allows enhanced and uniform drug distribution, optimized therapeutic indices, and greatly reduced systemic toxicity to patients. ${ }^{4-6}$

There is currently no standard method for evaluating treatment response at the CED infusion site that is different from evaluating the whole-tumor treatment response. In this study, we used MR spectroscopic imaging (MRSI), a noninvasive imaging technique that provides information on the tissue metabolites. ${ }^{7}$ Due to the unique metabolic characteristics of tumors, MRSI has been used to aid in diagnosing various cancers and evaluating intratumoral heterogeneity and treatment response in both ani-

Please address correspondence to Sunitha Thakur, PhD, 300 East 66th St, Breast Imaging Center, Memorial Sloan Kettering Cancer Center, New York, NY 10065; e-mail: thakurs@mskcc.org

http://dx.doi.org/10.3174/ajnr.A4713 
mals and humans. ${ }^{8}$ MRSI allows the analysis of many different metabolites in tumor and normal tissue regions of the brain. Some of the major metabolites of interest in examining brain tissue are choline compounds, creatine, and $N$-acetylaspartate. ${ }^{7,8}$ Cho represents the sum of choline compounds, which are components of cell membrane and increase with cellular proliferation. $\mathrm{Cr}$ is a biomarker of energy metabolism and is generally stable, functioning primarily as a standard comparison of the other metabolites, though it can vary within certain tumors. ${ }^{9}$ NAA is used as a biomarker for neuronal and axonal integrity and will decrease with neuronal damage or loss. The metabolite ratios of $\mathrm{Cho} / \mathrm{Cr}$ and $\mathrm{Cho} / \mathrm{NAA}$ were the parameters of interest in this study.

In the current descriptive study, we used multivoxel proton ${ }^{1} \mathrm{H}-\mathrm{MR}$ spectroscopy to compare metabolic changes at the CED infusion site with tumor outside the CED-infused area. We used voxels filled with dominant normal white matter as a reference. We hypothesized that multivoxel ${ }^{1} \mathrm{H}-\mathrm{MR}$ spectroscopy would show a difference in the change of metabolites at the CED-infused area compared with the non-CED infused area.

\section{MATERIALS AND METHODS \\ Participants}

We prospectively analyzed MR spectroscopy data from 6 patients diagnosed with DIPG who received CED of a therapeutic antibody in a Phase I clinical trial (NCT01502917), which was approved by the Memorial Sloan Kettering Cancer Center review board. The median age of patients was 7 years (range, 3-17 years). Patients were diagnosed with DIPG on the basis of clinical presentation and MR imaging findings. Before CED treatment, they all completed standard external beam radiation therapy (54-60 Gy) for at least 9 weeks, but for no more than 14 weeks. MR spectroscopic imaging was performed at $\geq 3$ time points for each patient: 1 week before treatment (preCED), 1 day after treatment (postCED1), and 1 month after treatment (postCED2). All patients were required to have imaging at 1 month after CED (postCED2). Some patients had additional scans at 1-month intervals afterward (postCED3 and 3 months after CED treatment) if they were able to return more frequently due to their close proximity or willingness to travel for additional scans.

\section{MRI/MRSI Methods}

The MR imaging/MRSI studies were performed by using a wholebody 3T Discovery MR750 scanner (GE Healthcare, Milwaukee, Wisconsin) with a quadrature head coil as the transceiver. The MR imaging protocol included precontrast sagittal and axial T1weighted, axial T2-weighted, axial T2 FLAIR, axial diffusionweighted, and postcontrast T1-weighted sequences in axial, sagittal, and coronal planes. The precontrast T2 FLAIR images were obtained by using a fast spin-echo sequence (TI/TE/TR $=2250$ / $120 / 9000 \mathrm{~ms}, \mathrm{FOV}=24 \mathrm{~cm})$. The $3-\mathrm{mm}$ section thickness with no spacing between sections $(320 \times 256$ acquisition matrix size $)$ served as scouts for placement of the volume of interest for the 3D MRSI data acquisition. MRSI data acquisition was performed following the precontrast MR imaging acquisition, but before gadolinium contrast administration. The 3D MRSI VOI encompassed the lesion (hyperintense area on T2 FLAIR images) and surround- ing normal-appearing brain tissue. Outer volume saturation bands were applied to avoid signal contaminations caused by subcutaneous lipid, bone, and varying magnetic susceptibility effects that might compromise the quality of the spectra. A point-resolved spectroscopy sequence with water suppression by using chemical shift selective suppression was used to collect the $3 \mathrm{D}$ ${ }^{1} \mathrm{H}$-MRSI datasets with TE $=144 \mathrm{~ms}$, TR $=1000 \mathrm{~ms}$, FOV $=8$ $\mathrm{cm}$, section thickness $=10 \mathrm{~mm}, 3 \mathrm{D}$ spatial-encoding $(8 \times 8 \times 8$ matrix size, resulting in $1-\mathrm{cm}^{3}$ nominal MRSI voxel size), $5000-\mathrm{Hz}$ spectral width, and 4096 data points. The acquisition time for 3D MRSI was approximately 9 minutes, including the prescan for magnetic field shimming. The MRSI VOI location, size, and acquisition parameters were kept the same for each patient during follow-up studies by using anatomic landmarks. The axial postcontrast T1WI MR images were collected with the same section number, location, and thickness as the axial T2 FLAIR images.

\section{MR Spectroscopy Data Processing}

Multivoxel ${ }^{1} \mathrm{H}-\mathrm{MR}$ spectroscopy data were analyzed with FuncTool software (Advantage Workstation, Version 11.3-4.2; GE Healthcare). The MRSI sections were interpolated to $3 \mathrm{~mm}$ to match the FLAIR images and generate overlay images of the MRSI grid onto the corresponding T2-FLAIR image. ROIs were equivalent to 1 MR spectroscopy voxel (nominal size of 1 cubic centimeter) and were selected by using the overlaid T2-FLAIR images for anatomic reference. Major resonance peaks of NAA (2.0 ppm), Cr (3.0 ppm), and Cho (3.2 ppm) for each MRSI voxel were assigned and numerically integrated to estimate peak areas. Peak area ratios were calculated and color maps were generated for Cho/NAA and Cho/Cr.

\section{ROI Selection}

Several ROIs were chosen to analyze MR spectroscopic data: a voxel at the CED infusion, a voxel that was within the tumor but outside the CED infusion, additional voxels that were within the tumor to calculate the average tumor metabolites, and normal tissue voxels as controls. A radiologist with a Certificate of Added Qualification in radiology (S.H., 11 years of experience) and a physicist (S.B.T., 12 years of experience in brain MR spectroscopy) oversaw all ROI voxel selections. The CED infusion voxel was selected by using the postCED1 scans because the infusion site was visible as hyperintense signal on the T2-FLAIR images (Fig $1 A-D)$. The CED infusion site voxels for preCED, postCED2, and, when applicable, postCED3 were selected by matching the section numbers and location by using anatomic landmarks to the postCED1 infusion site voxel. The tumor non-CED infusion site voxel was chosen at least 2 sections away from the CED infusion site and was verified by the radiologist (S.H.) to be outside of the CED infused volume of distribution (Fig $1 E-H$ ).

Color metabolite maps were used to observe the relative intensity of each metabolite and metabolite ratio in and around the CED infusion site. Anatomic landmarks and section numbers were used to best match equivalent tumor non-CED infusion site voxels for the remaining scans. Additional tumor voxels were selected on the basis of MR imaging and spectra that were characteristic of tumor (high Cho and low NAA peak compared with unaffected tissue and metabolite ratios: $\mathrm{Cho} / \mathrm{Cr}$ of $>1.5$ and Cho/ 


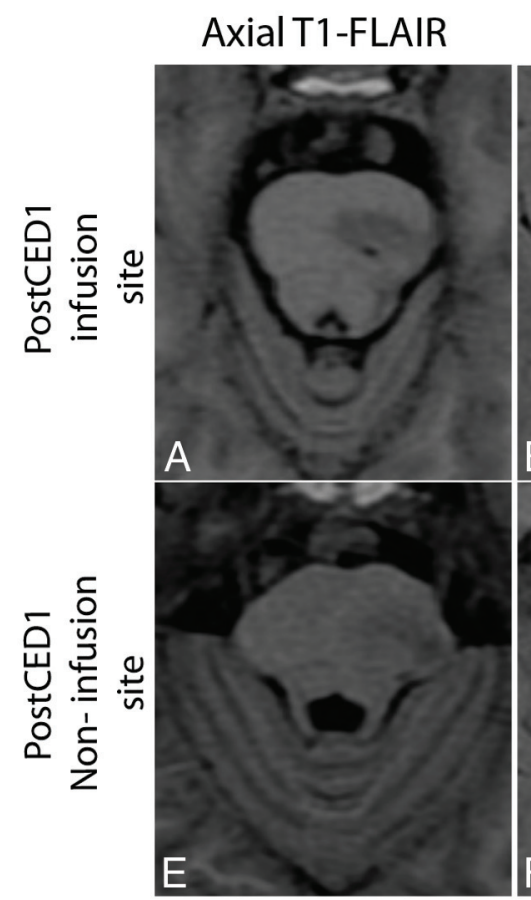

Axial T2-FLAIR

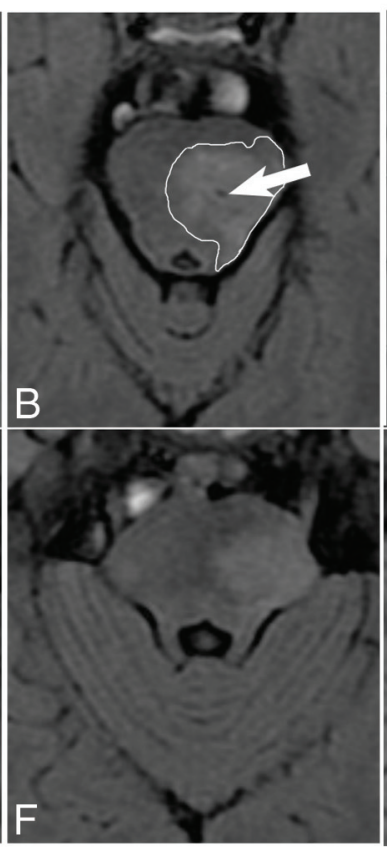

${ }^{1} \mathrm{H}-\mathrm{MRS} 2 \mathrm{D}$ Slice

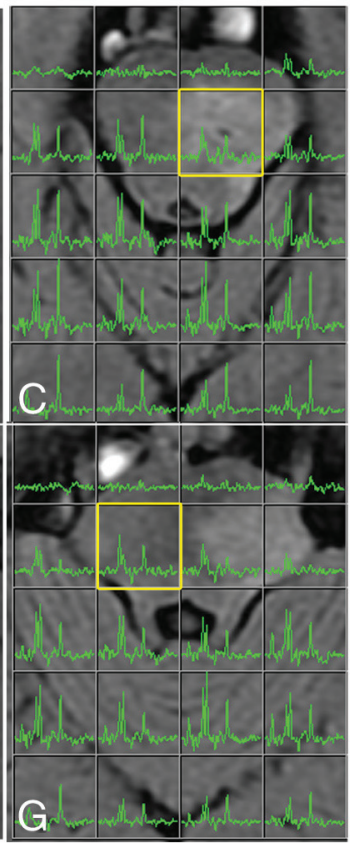

${ }^{1} \mathrm{H}-\mathrm{MRS}$ spectra

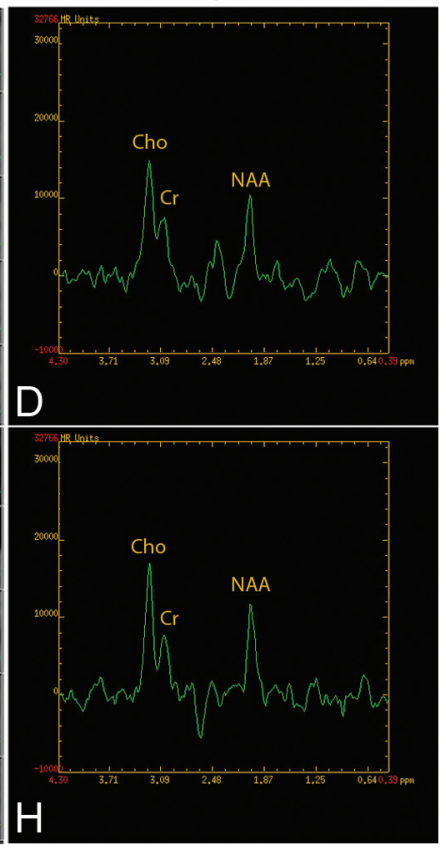

FIG 1. ROI selection. A, Axial T1-weighted FLAIR image shows regional hypointensity at the CED infusion site. $B$, The CED infusion site (white arrow) is also visible on the axial T2 FLAIR image with surrounding T2-hyperintense signal (outlined in white) indicative of the CED infusion volume of distribution. C, The corresponding axial T2-FLAIR with multivoxel MR spectroscopy is shown with the selected CED infusion site voxel (yellow box). D, Enlarged view of the MR spectra for the selected CED infusion site voxel. $E$ and $F$, The axial T1-weighted FLAIR and axial T2-FLAIR images of the noninfusion site section. $G$, The selected tumor non-CED infusion site voxel (yellow box) is outside the CED infusion volume of distribution. $H$, The enlarged view of the MR spectra for the selected tumor non-CED infusion site voxel.

NAA of $>1$ ). An average of the tumor metabolite ratios was calculated for each scan by taking an average of the Cho/Cr and Cho/NAA ratios from the following voxels of tumor: the CED infusion site, the tumor non-CED infusion site, and the other additional tumor voxels. Normal tissue voxels were chosen in the white matter cerebellum or cerebrum away from the tumor and had spectra characteristic of normal white matter with metabolite ratios of Cho/Cr of $\sim 0.7-1$ and Cho/NAA of $\sim 0.5-1 .{ }^{10-12}$

\section{Statistical Analysis}

Statistical analysis was performed on metabolite ratios, which were treated as data with unknown distributions. Exploratory data analysis included group means/medians with time and graphing individual measurements with time. To test the trend of changes in metabolite ratios, we performed paired difference tests by using the Wilcoxon signed rank test in $\mathrm{R}$ (http://www. r-project.org).

\section{RESULTS}

The metabolite ratios Cho/Cr and Cho/NAA for the CED infusion site, tumor non-CED infusion site, average tumor, and normaltissue ROIs were compared during the course of treatment. Color metabolite maps demonstrated the higher intensity of Cho, Cho/ $\mathrm{Cr}$, and Cho/NAA and lower intensity of NAA in the area of the pons with tumor (Fig 2).

The metabolite ratios for normal tissue remained stable and within the normal range for each patient throughout the course of treatment (Fig 3). In 4 patients, Cho/Cr and Cho/ NAA decreased at the CED infusion site with time, while the ratios for the tumor non-CED infusion site generally decreased by a lesser degree or increased with time (Fig $4 A-D$ ). In 2 patients, Cho/Cr and Cho/NAA increased at the infusion site with time, but a more substantial increase occurred in $\mathrm{Cho} / \mathrm{Cr}$ and Cho/NAA at the tumor non-CED infusion site and for the average tumor (Fig $4 E,-F$ ). In 2 patients, Cho/Cr and Cho/ NAA in the CED infusion site ROI at postCED1 were high (Fig $4 B,-E)$.

Statistical analysis was performed to assess the change in Cho/Cr and Cho/NAA at the CED infusion site compared with the tumor non-CED infusion site in all patients (Fig 5). There was a relative difference in the percentage change in both $\mathrm{Cho/Cr}$ and Cho/NAA for the CED infusion site compared with the tumor non-CED infusion site. The mean and $P$ value decreased for both $\mathrm{Cho} / \mathrm{Cr}$ and $\mathrm{Cho} / \mathrm{NAA}$ metabolite ratios with time when comparing preCED with postCED1, preCED with postCED2, and preCED with postCED3. The mean (SD) for the change in Cho/Cr between infusion and non-CED infusion sites was similar between postCED1 and postCED2 compared with preCED: 1.20 (0.53) and 1.21 (0.43), respectively; but it decreased at postCED3 to $0.66(0.06)$. The mean (SD) change in Cho/NAA between the infusion and non-CED infusion sites decreased from 1.11 (0.66) to $0.93(0.97)$ to $0.82(0.29)$ at postCED1, postCED2, and postCED3, respectively. The $P$ value for the change in $\mathrm{Cho} / \mathrm{Cr}$ at the CED infusion site compared with the tumor non-CED infusion site decreased from 0.84 at postCED1 to 0.25 at postCED3. The $P$ value for the change in Cho/NAA decreased from 1.00 at postCED1 to 0.50 at postCED3. 


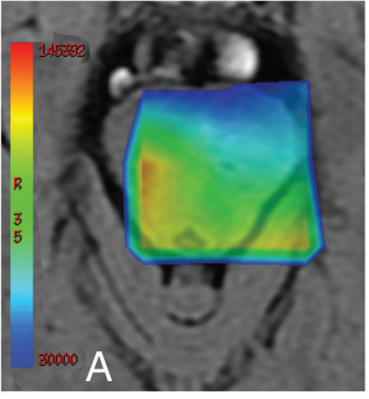

NAA

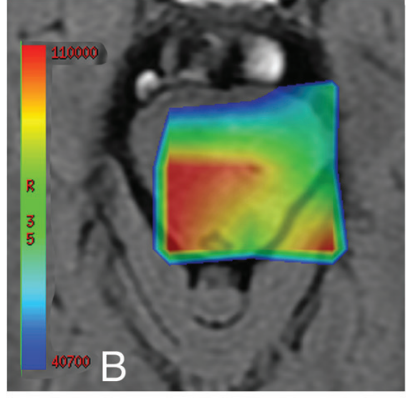

Cho

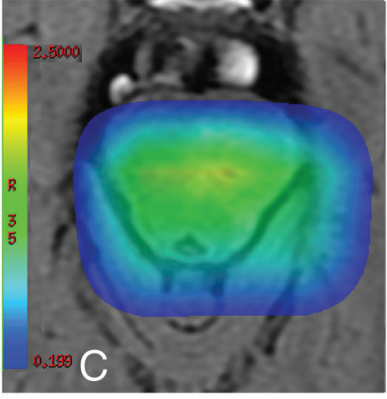

$\mathrm{Cho} / \mathrm{Cr}$

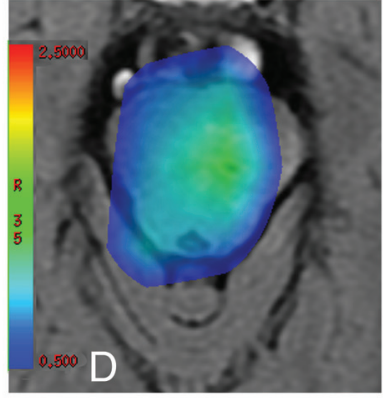

Cho/NAA

FIG 2. Color metabolite maps. Relative intensities of metabolites NAA $(A), C h o(B), C h o / C r(C)$, and Cho/NAA $(D)$ are shown in this postCEDI infusion section. The CED infusion site is visible and is located in the area of the pons with a higher intensity signal of $\mathrm{Cho}, \mathrm{Cho} / \mathrm{Cr}$, and $\mathrm{Cho} / \mathrm{NAA}$ and lower NAA intensity.
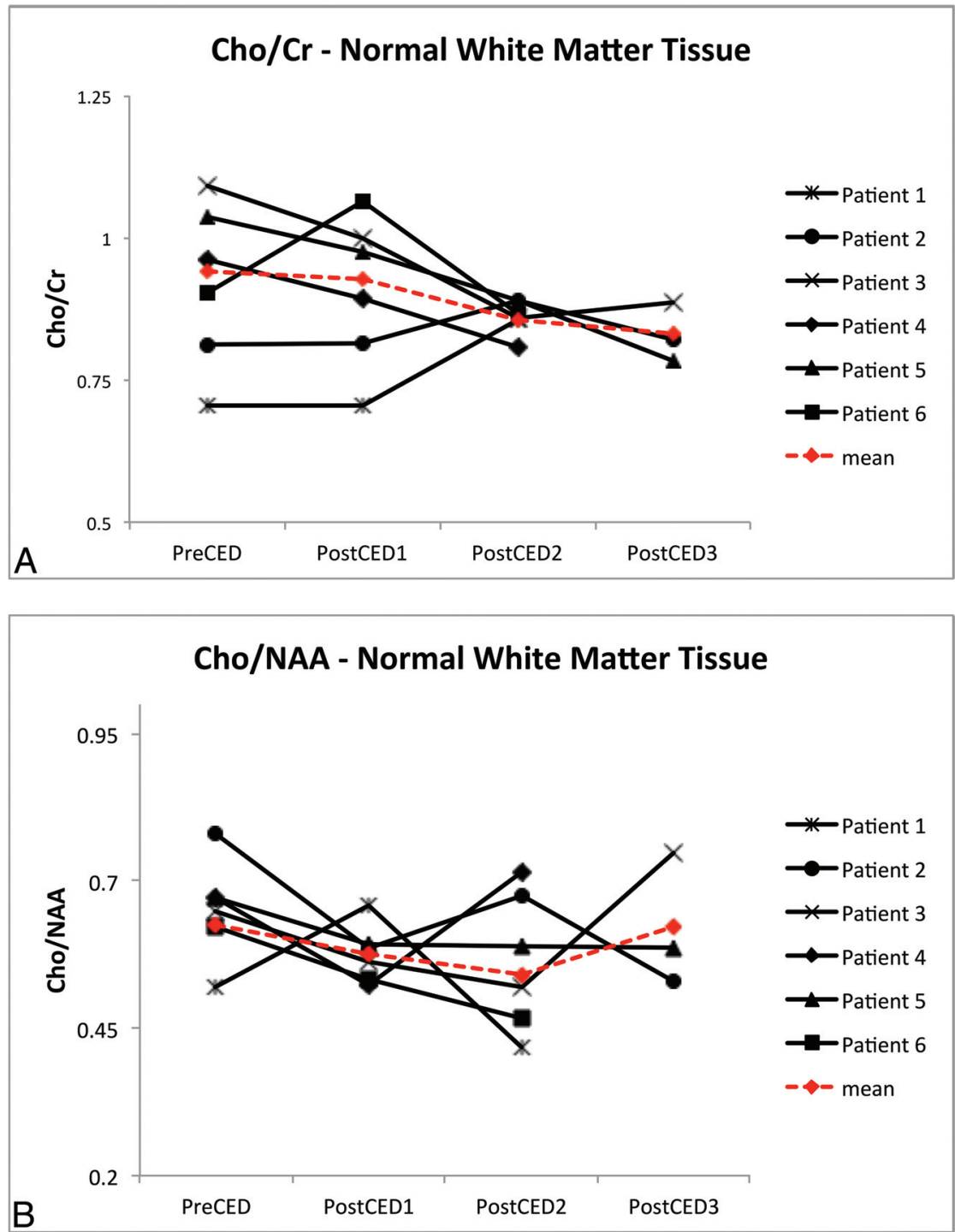

FIG 3. Metabolite ratios for normal white matter. $\mathrm{Cho} / \mathrm{Cr}(A)$ and Cho/NAA (B) ratios for normal cerebellar or cerebral white matter for each patient are shown throughout the course of treatment, and the mean value at each time point is shown in red. Both metabolite ratios for each patient remained stable and in the expected normal range.

\section{DISCUSSION}

Despite much therapeutic effort, patients with DIPG continue to have a poor prognosis with a median survival of $<1$ year. Radia- tion therapy is the standard of care because most chemotherapeutic agents do not prolong survival. CED is a promising technique that allows direct localized delivery of therapeutic agents to the tumor. In this study, patients with nonprogressive DIPG received CED treatment with a therapeutic antibody in a Phase I clinical trial (NCT01502917). Evaluating local treatment response at the CED infusion site is critical because it may suggest that there is some treatment effect when whole-tumor response may be undetectable. A CED therapeutic agent may be falsely deemed ineffective if whole-tumor response is assessed without evaluating the response at the local infusion site.

Evaluating DIPG treatment response with conventional MR imaging alone is inadequate due to nonspecific changes on imaging that can arise from recurrent tumor, inflammatory responses, and necrotic changes from treatment. ${ }^{12,13}$ Furthermore, conventional MR imaging results alone have not been shown to correlate with survival in patients with DIPG. ${ }^{14}$ Diffusion tensor imaging and diffusion-weighted imaging have been studied in patients with DIPG, but the diffuse infiltration of DIPG has made using these techniques difficult. The pattern and amount of tract involvement varies among patients, and the significance of diffusion properties in DIPG varies anatomically. ${ }^{15,16}$ Perfusion-weighted MR imaging has been used to assess the angiogenesis of the tumor, but relative tumor blood volume has a weaker correlation to disease progression than increased choline levels with ${ }^{1} \mathrm{H}-\mathrm{MR}$ spectroscopy. ${ }^{17}$ 

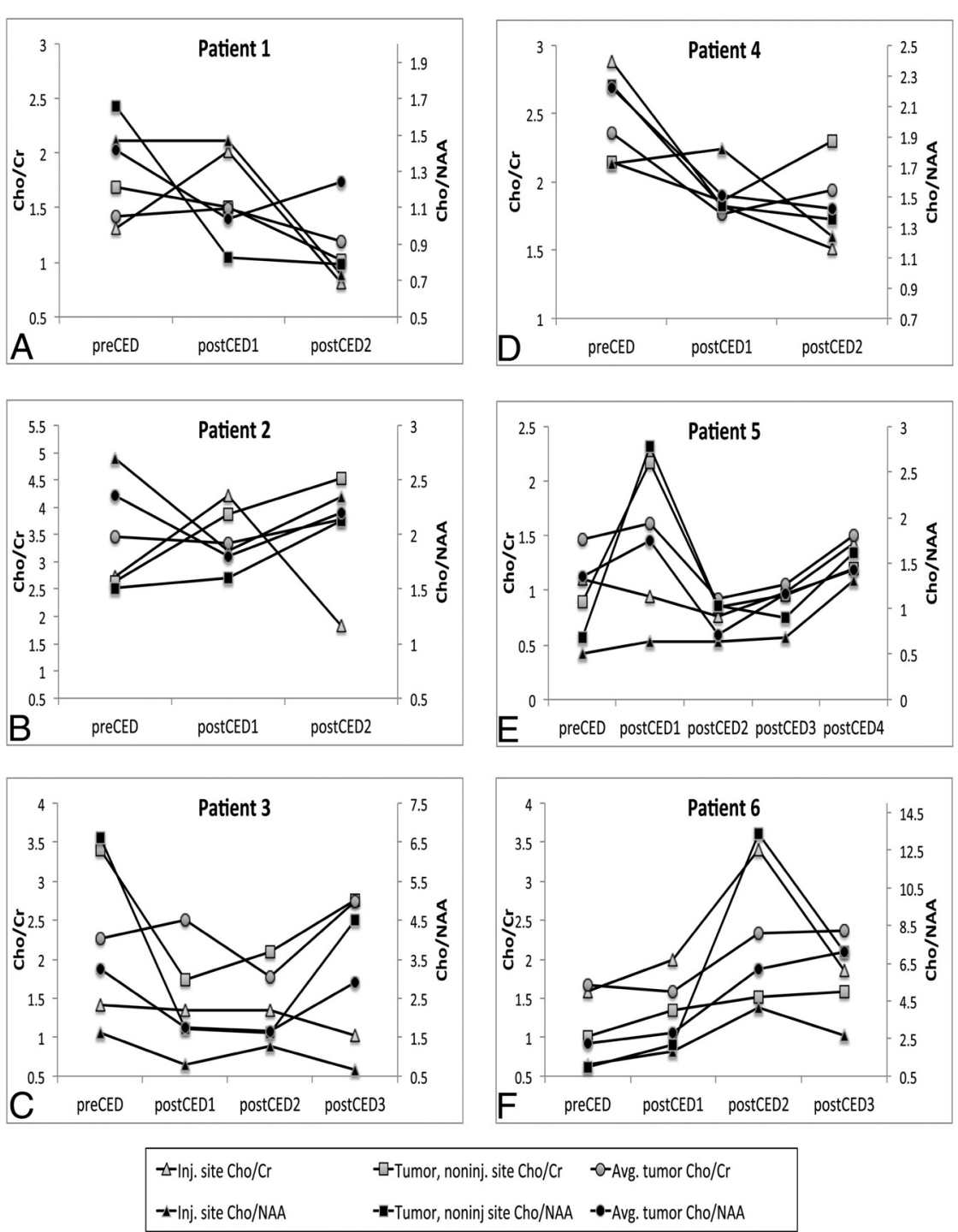

FIG 4. Metabolite ratios for CED infusion site, tumor non-CED infusion site, and average tumor for individual patients. $A-D$, Patients $1-4$ show a similar trend with a comparative decrease in $\mathrm{Cho} / \mathrm{Cr}$ and Cho/NAA with time for the CED infusion site compared with the tumor non-CED infusion site and average tumor voxels. $E$ and $F$, Patients 5 and 6 show a slight increase in $\mathrm{Cho} / \mathrm{Cr}$ and Cho/NAA for the CED infusion site and a comparatively larger increase in both ratios for the tumor non-CED infusion site and the average tumor.

${ }^{1} \mathrm{H}-\mathrm{MR}$ spectroscopy is effective in providing clinically important information in brain tumors and has increased diagnostic sensitivity, specificity, and accuracy when used with MR imaging. ${ }^{8} \mathrm{H}-\mathrm{MR}$ spectroscopy uses the radiofrequency signal from protons when a magnetic field is applied to determine metabolite concentrations by displaying a spectrum of peaks corresponding to different metabolites. The area under the curve of the peak is then used to calculate the concentration and ratios of the metabolites. ${ }^{7}$ Due to the abnormal metabolism characteristic of tumor cells, MR spectroscopy can be used to identify tumorous tissue with good accuracy. Cho, Cr, and NAA are some of the most common metabolites analyzed for brain tumors. Increases in Cho correspond to increased membrane turnover or cellular density and thus make Cho a biomarker for tumor tissue. ${ }^{7} \mathrm{Cr}$ is usually stable because it is a biomarker of energy metabolism, but it can vary within certain tumors, and a decrease in Cr can signify tissue necrosis. ${ }^{7,9}$ Decreasing levels of NAA signify a decrease in neuronal integrity. ${ }^{7}$ $\mathrm{Cho/Cr}$ and Cho/NAA ratios have often been used to identify the metabolic changes in tumor tissue. ${ }^{1,12}$ Increases in both Cho/Cr and Cho/NAA indicate tumor because Cho increases, Cr generally stays stable or decreases, and NAA decreases in tumor tissue.

Single-voxel ${ }^{1} \mathrm{H}-\mathrm{MR}$ spectroscopy has been used to assess prognosis and overall treatment response to radiation therapy and chemotherapeutics in DIPG tumors. ${ }^{8,18,19}$ However, multivoxel ${ }^{1} \mathrm{H}$-MR spectroscopy allows the interrogation of local changes in metabolism within different regions of the tumor. ${ }^{13}$ A previous study used multivoxel ${ }^{1} \mathrm{H}-\mathrm{MR}$ spectroscopy to look at DIPG during radiation therapy and compared changes in tumor versus normal brain tissue. This study showed a correlation with low Cho/Cr and Cho/NAA during response to radiation therapy and high Cho/Cr and Cho/NAA with relapse. ${ }^{12}$ Other studies have also shown increases in Cho/Cr and Cho/NAA ratios in DIPG tumors corresponding to prognosis and relapse after treatment. ${ }^{20,21}$ In our study, we wanted to evaluate the utility of using multivoxel ${ }^{1} \mathrm{H}-\mathrm{MR}$ spectroscopy for assessing CED treatment. We prospectively analyzed the multivoxel ${ }^{1} \mathrm{H}$-MR spectroscopy data from 6 patients with DIPG receiving CED treatment. We compared the Cho/Cr and Cho/NAA ratios across time at the CED infusion site with the tumor nonCED infusion site outside the CED infused volume of distribution.

Based on the MR spectroscopy data for these patients, there is a comparative reduction in $\mathrm{Cho} / \mathrm{Cr}$ and $\mathrm{Cho} / \mathrm{NAA}$ after $\mathrm{CED}$ treatment at the CED infusion site compared with the tumor non-CED infusion site. This reduction becomes more pronounced with increasing time after CED treatment. These MR spectroscopic changes, when combined with findings from other modalities, such as DWI, DTI, PWI, and PET and compared with patient outcomes, may reflect CED treatment response. In 2 patients, metabolite ratios for the CED infusion site ROI at postCED1 were high compared with their baseline metabolite ratios at preCED. Patient 2 had Cho/Cr $=4.22$ for the CED infusion site and 3.88 for the tumor noninfusion site, respectively, at postCED1 (Fig 4B). Patient 5 had Cho/NAA $=2.78$ and $\mathrm{Cho} / \mathrm{Cr}=$ 2.17 for the tumor noninfusion site at postCED1 (Fig $4 E$ ). Because postCED1 is 1 day after CED treatment, there may be an influence of the infused CED volume on the MR spectroscopic readings. The infusate may produce susceptibility arti- 


\section{Change in $\mathrm{Cho} / \mathrm{Cr}$ from baseline: Infused/ Non-infused}

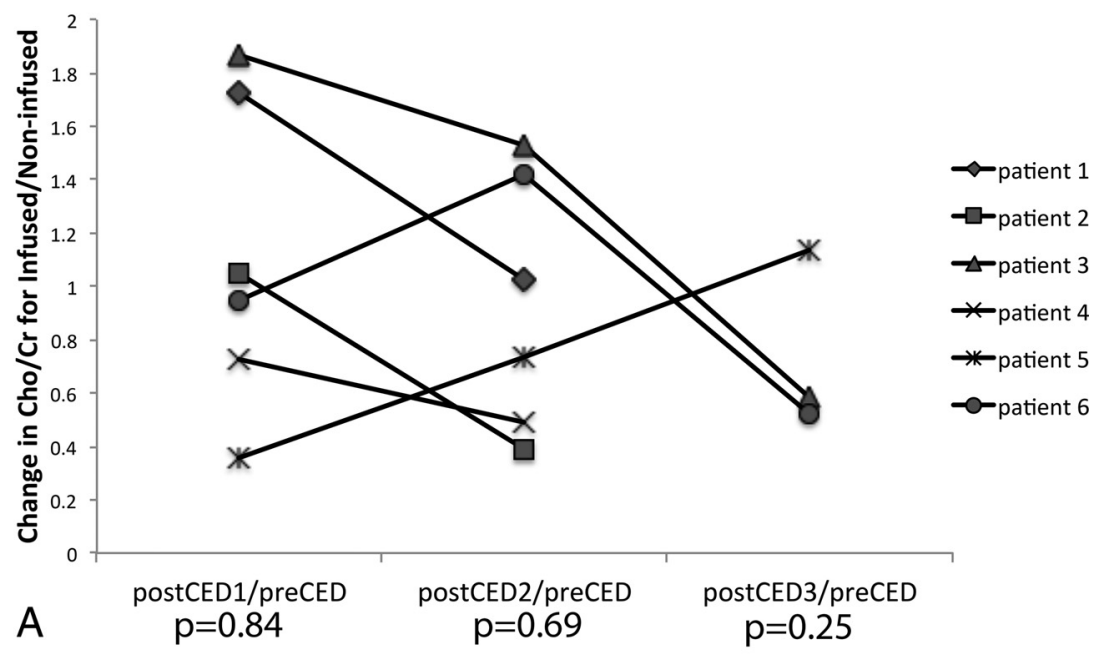

Change in Cho/NAA from baseline: Infused/ Non-infused

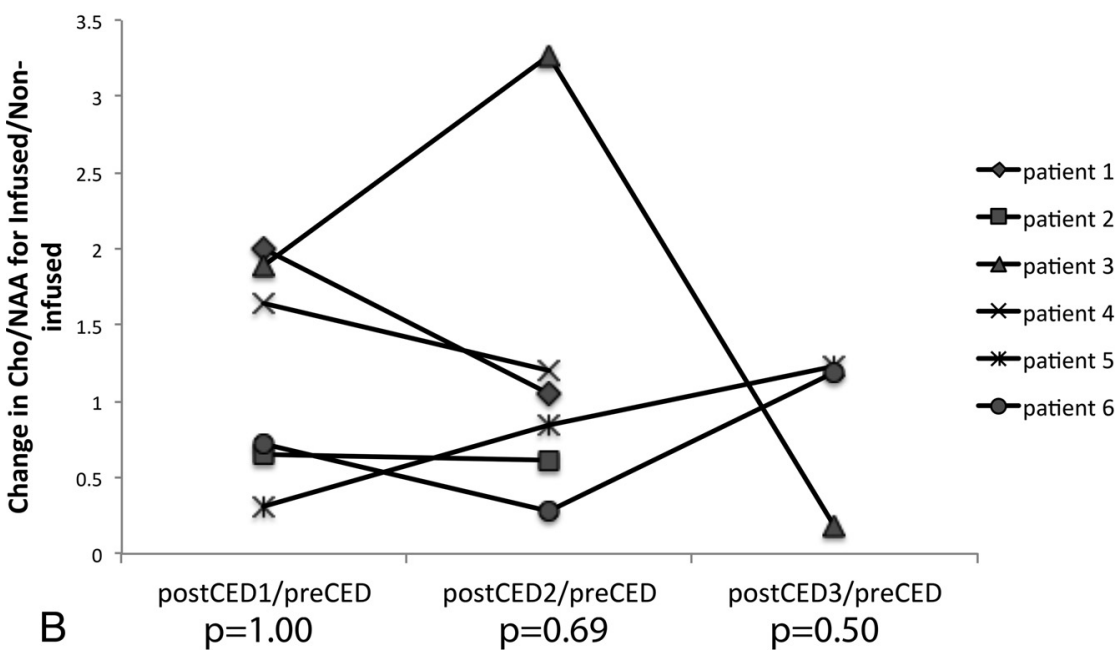

FIG 5. CED infusion site versus tumor non-CED infusion site $\mathrm{Cho} / \mathrm{Cr}$ and $\mathrm{Cho/NAA}$ comparison. The change in metabolite ratios $\mathrm{Cho} / \mathrm{Cr}(A)$ and $\mathrm{Cho} / \mathrm{NAA}(B)$ from the baseline preCED scan to postCED1, postCED2, and postCED3 scans is calculated for the group of patients at both the CED infusion site and tumor non-CED infusion site. The change in Cho/ $\mathrm{Cr}$ and $\mathrm{Cho} / \mathrm{NAA}$ at the CED infusion site compared with the tumor non-CED infusion site is calculated by using the Wilcoxon signed rank test. While no statistical claims can be made, the decreasing $P$ values for both metabolite ratios suggest that a comparative reduction in the ratios becomes more pronounced with time.
preCED scan. These variables may influence the response to CED treatment. It may be of interest for future studies to derive absolute measures of metabolites that could provide an accurate way to study the local changes with treatment and to standardize the patient's tumor size and baseline metabolic ratios.

\section{CONCLUSIONS}

Our methodology for analysis of multivoxel ${ }^{1} \mathrm{H}-\mathrm{MR}$ spectroscopy highlighted a trend of decreasing $P$ values for the metabolite ratios $\mathrm{Cho} / \mathrm{Cr}$ and Cho/NAA after CED treatment at the CED infusion site compared with the tumor non-CED infusion site. Although statistical tests were not significant with the small number of patients, the results are encouraging, and if confirmed, MRSI could be used to obtain early markers of tumor response in individual patients. Our methodology may also be applicable to other imaging modalities, with some optimization for determining ROI size and placement. When combined with other imaging techniques, such as PET, DTI, DWI, and PWI, our methodology may provide a clinical tool to more accurately evaluate local tumor response after CED treatment.

Disclosures: Mark M. Souweidane-UNRELATED Consultancy: Aesculap Neuro-Endoscopy Advisory Council; Payment for Lectures (including service on Speakers Bureaus): Aesculap NeuroEndoscopy Advisory Council, Comments: annual endoscopic practical course; Travel/Accommodations/Meeting Expenses Unrelated to Activities Listed: Aesculap Neuro-Endoscopy Advisory Council, Comments: annual endoscopic practical course. facts that could result in poor shimming and hence broader line widths. This result could lead to inaccurate quantification of metabolites at postCED1. Despite this limitation, we were able to see a difference in metabolite ratios at the infusion site versus the noninfused tumor by using our methodology with multivoxel ${ }^{1} \mathrm{H}$-MR spectroscopy. Our methodology for ROI selection to assess CED treatment response can also be used in parallel to analyze results from other imaging modalities such as DWI and PWI, which may provide additional biomarkers to help evaluate the response in CED treatment.

The study is limited in that a reduced metabolite ratio trend without statistical significance was observed on the basis of the small number of patients analyzed with only a few scans. Additionally, the patients started with different tumor volumes and with different metabolite ratios from their baseline

\section{REFERENCES}

1. Jansen MH, van Vuurden DG, Vandertop WP, et al. Diffuse intrinsic pontine gliomas: a systematic update on clinical trials and biology. Cancer Treat Rev 2012;38:27-35 CrossRef Medline

2. Warren KE. Diffuse intrinsic pontine glioma: poised for progress. Front Oncol 2012;2:205 CrossRef Medline

3. Jain RK. The next frontier of molecular medicine: delivery of therapeutics. Nat Med 1998;4:655-57 CrossRef Medline

4. Sandberg DI, Edgar MA, Souweidane MM. Convection-enhanced delivery into the rat brainstem. J Neurosurg 2002;96:885-91 CrossRef Medline

5. Song DK, Lonser RR. Convection-enhanced delivery for the treatment of pediatric neurologic disorders. J Child Neurol 2008;23: 1231-37 CrossRef Medline

6. Bobo RH, Laske DW, Akbasak A, et al. Convection-enhanced delivery of macromolecules in the brain. Proc Natl Acad Sci U S A 1994; 91:2076-80 CrossRef Medline 
7. Gujar SK, Maheshwari S, Björkman-Burtscher I, et al. Magnetic resonance spectroscopy. J Neuroophthalmol 2005;25:217-26 CrossRef Medline

8. Glunde KB, Bhujwalla ZM. Metabolic tumor imaging using magnetic resonance spectroscopy. Semin Oncol 2011;38:26-41 CrossRef Medline

9. Kinoshita Y, Yokota A. Absolute concentrations of metabolites in human brain tumors using in vitro proton magnetic resonance spectroscopy. NMR Biomed 1997;10:2-12 Medline

10. Soares DP, Law M. Magnetic resonance spectroscopy of the brain: review of metabolites and clinical applications. Clin Radiol 2009;64: 12-21 CrossRef Medline

11. Howe FA, Barton SJ, Cudlip SA, et al. Metabolic profiles of human brain tumors using quantitative in vivo $1 \mathrm{H}$ magnetic resonance spectroscopy. Magn Reson Med 2003;49:223-32 CrossRef Medline

12. Laprie A, Pirzkall A, Haas-Kogan DA, et al. Longitudinal multivoxel MR spectroscopy study of pediatric diffuse brainstem gliomas treated with radiotherapy. Int J Radiat Oncol Biol Phys 2005;62: 20-31 CrossRef Medline

13. Yang I, Huh NG, Smith ZA, et al. Distinguishing glioma recurrence from treatment effect after radiochemotherapy and immunotherapy. Neurosurg Clin N Am 2010;21:181-86 CrossRef Medline

14. Hargrave D, Chuang N, Bouffet E. Conventional MRI cannot predict survival in childhood diffuse intrinsic pontine glioma. J Neurooncol 2008;86:313-19 CrossRef Medline

15. Helton KJ, Phillips NS, Khan RB, et al. Diffusion tensor imaging of tract involvement in children with pontine tumors. AJNR Am J Neuroradiol 2006;27:786-93 Medline

16. Chen HJ, Panigrahy A, Dhall G, et al. Apparent diffusion and fractional anisotropy of diffuse intrinsic brain stem gliomas. AJNR AmJ Neuroradiol 2010;31:1879-85 CrossRef Medline

17. Tzika AA, Astrakas LG, Zarifi MK, et al. Spectroscopic and perfusion magnetic resonance imaging predictors of progression in pediatric brain tumors. Cancer 2004;100:1246-56 CrossRef Medline

18. Meisamy S, Bolan PJ, Baker EH, et al. Neoadjuvant chemotherapy of locally advanced breast cancer: predicting response with in vivo (1)H MR spectroscopy: a pilot study at 4 T. Radiology 2004;233: 424-31 CrossRef Medline

19. Steffen-Smith EA, Venzon DJ, Bent RS, et al. Single- and multivoxel proton spectroscopy in pediatric patients with diffuse intrinsic pontine glioma. Int J Radiat Oncol Biol Phys 2012;84:774-79 CrossRef Medline

20. Laprie A, Catalaa I, Cassol E, et al. Proton magnetic resonance spectroscopic imaging in newly diagnosed glioblastoma: predictive value for the site of postradiotherapy relapse in a prospective longitudinal study. Int J Radiat Oncol Biol Phys 2008;70:773-81 CrossRef Medline

21. Hipp SJ, Steffen-Smith E, Hammoud D, et al. Predicting outcome of children with diffuse intrinsic pontine gliomas using multiparametric imaging. Neuro Oncol 2011;13:904-09 CrossRef Medline 